\title{
Theory and laboratory experiments of elastic wave scattering by dry planar fractures
}

\author{
Thomas E. Blum, ${ }^{1}$ Roel Snieder, ${ }^{2}$ Kasper van Wijk, ${ }^{1}$ and Mark E. Willis ${ }^{3}$ \\ Received 8 February 2011; revised 25 May 2011; accepted 3 June 2011; published 30 August 2011.
}

[1] Remote sensing of fractures with elastic waves is important in fields ranging from seismology to nondestructive testing. In many geophysical applications, fractures control the flow of fluids such as water, hydrocarbons or magma. While previous analytic descriptions of scattering mostly deal with very large or very small fractures (compared to the dominant wavelength), we present an analytic solution for the scattering of elastic waves from a fracture of arbitrary size. Based on the linear slip model for a dry fracture, we derive the scattering amplitude in the frequency domain under the Born approximation for all combinations of incident and scattered wave modes. Our analytic results match laser-based ultrasonic laboratory measurements of a single fracture in clear plastic, allowing us to quantify the compliance of a fracture.

Citation: Blum, T. E., R. Snieder, K. van Wijk, and M. E. Willis (2011), Theory and laboratory experiments of elastic wave scattering by dry planar fractures, J. Geophys. Res., 116, B08218, doi:10.1029/2011JB008295.

\section{Introduction}

[2] Faults and fractures in the subsurface can act as conduits or barriers to fluid flow of hydrocarbons, water and magma [Haney et al., 2005; Brandsdóttir and Einarsson, 1979]. Changes in fracturing lead to changes in coda waves as well as attenuation and seismic anisotropy. Collettini et al. [2009] show that the mechanical properties of fractures and fault zones are related to the fabric and microstructure of these features. Understanding the interaction of fractures with elastic waves is crucial in order to characterize fracture properties remotely. In hydrocarbon reservoirs, hydraulic fractures are generated to stimulate production and can be monitored with active or passive sources [Wills et al., 1992; Meadows and Winterstein, 1994]. Moreover, scattered waves can be used as a tool for monitoring fracture growth and fracture evolution [Groenenboom and Fokkema, 1998; Groenenboom and van Dam, 2000; Pyrak-Nolte, 2000]. Besides geophysical applications, scattering from fractures is important in nondestructive testing applications [Langenberg et al., 2002].

[3] Gubernatis et al. [1977a] derive the general integral equation for an elastic scatterer, which they solve using the Born approximation [Gubernatis et al., 1977b; Wu and Aki, 1985]. Their work is based on a volumetric flaw with specified contrast in density and elastic properties. In contrast, the linear slip model handles planar fractures of negligible aperture by linking the discontinuity of the displacement

\footnotetext{
${ }^{1}$ Physical Acoustics Laboratory, Department of Geosciences, Boise State University, Boise, Idaho, USA.

${ }^{2}$ Center for Wave Phenomena, Colorado School of Mines, Golden, Colorado, USA.

${ }^{3}$ ConocoPhillips Company, Houston, Texas, USA.

Copyright 2011 by the American Geophysical Union. 0148-0227/11/2011JB008295
}

field at the fracture plane to the traction at the slip interface [Schoenberg, 1980]. This model can be directly applied to fractures with a size comparable to the wavelength. The extreme case where the fracture plane is infinite leads to frequency dependent reflection and transmission coefficients [Pyrak-Nolte et al., 1990; Pyrak-Nolte and Nolte, 1992; Zhu and Snieder, 2002]. The linear slip model is often used to describe dry fractures [Coates and Schoenberg, 1995], and can also be used for fluid-filled fractures [ $W u$ et al., 2005; Groenenboom and Falk, 2000]. It was also investigated experimentally [Pyrak-Nolte et al., 1992, 1996]. In addition, Sánchez-Sesma and Iturrarán-Viveros [2001] use the Sommerfeld optical diffraction theory to derive an approximate analytic expression for the scattering of $S H$ waves by a planar fracture of finite width and infinite length. Fang et al. [2010] present finite difference numerical simulations of the scattering of $P$ waves by a finite circular fracture.

[4] For multiple sets of parallel fractures of a small size compared to the dominant wavelength, wave propagation can be expressed in terms of effective medium theory [Crampin, 1981; Hudson, 1981; Schoenberg and Sayers, 1995; Schoenberg and Douma, 1988; Kachanov and Sevostianov, 2005]. This theory accounts for an effective velocity and attenuation across many parallel slip interfaces. Pyrak-Nolte et al. [1990] show that waves in such a medium are dispersive in nature and present laboratory anisotropy measurements in agreement with effective medium theory.

[5] Here, we apply the linear slip model to a single finite planar fracture under the Born approximation. From this, we develop an analytic expression for the general scattering amplitude without making assumptions about the fracture size or wavelength, and therefore are not restricted to small scatterers as used in earlier studies [e.g., Gubernatis et al., 1977b; Smyshlyaev and Willis, 1994]. We derive expres- 


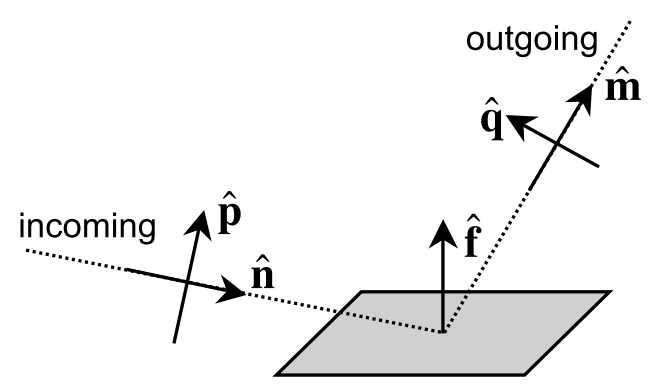

Figure 1. Definition of the normal vector $\hat{\mathbf{f}}$ to the fracture (shaded), the directions $\hat{\mathbf{n}}$ and $\hat{\mathbf{m}}$ of the incoming wave and outgoing waves, respectively. These vectors are also the polarization vectors in case of $P$ waves. For $S$ waves the polarization vectors of incoming and outgoing waves are $\hat{\mathbf{p}}$ and $\hat{\mathbf{q}}$, respectively.

sions for the scattering amplitude in the frequency domain for every combination of incoming and scattered body wave modes. We illustrate this theoretical work with a novel laboratory experiment by estimating the components of the compliance for a single crack generated in a clear plastic sample, and show that the measured scattering amplitude is explained by values of the compliance that are consistent with values reported in other studies.

\section{General Expressions for Scattering by a Fracture}

[6] We present the derivation in this paper in a frequency domain formulation based on the following Fourier convention: $f(t)=\int F(\omega) e^{-i \omega t} d \omega$. For brevity, we do not make the frequency dependence explicit, and use the Einstein summation convention. We first derive a general expression of the wave scattered by a fracture of arbitrary size. The stress across the fracture is continuous, but the displacement across the fracture is not necessarily continuous. We denote the discontinuity in the displacement by $[\mathbf{u}]$. According to Aki and Richards [2002, equation (3.2)], the displacement at location $\mathbf{x}$ due to the discontinuity of the displacement at the fracture $\Sigma$ is given by

$$
u_{n}(\mathbf{x})=\iint_{\Sigma}\left[u_{i}(\mathbf{s})\right] c_{i j k l} f_{j} G_{n k, l}(\mathbf{x}, \mathbf{s}) d^{2} s
$$

where $\iint_{\Sigma}(\cdots) d^{2} s$ denotes the integration over the surface of the fracture, $\hat{\mathbf{f}}$ is the normal vector to the fracture as shown in Figure 1, $c_{i j k l}$ is the elasticity tensor, and $G_{n k, l}$ is the gradient of the displacement Green's function defined as

$$
G_{n k, l}(\mathbf{x}, \mathbf{s})=\frac{\partial G_{n k}(\mathbf{x}, \mathbf{s})}{\partial s_{l}}
$$

[7] We next relate the discontinuity in the displacement to the stress field. We follow Schoenberg [1980] and assume that the slip discontinuity is related to the traction $\mathbf{T}$ at the fracture by a compliance matrix $\eta$ :

$$
\left[u_{i}\right]=\eta_{i r} T_{r} .
$$

Although this approximation may break down toward the edges of the fracture, it is commonly used in geophysics and considered accurate in far field [Wu et al., 2005]. Expressing the traction in the stress $\sigma_{i j}$ and the normal vector to the fracture yields

$$
\left[u_{i}\right]=\eta_{i r} \sigma_{r s} f_{s}
$$

hence

$$
\left[u_{i}\right] c_{i j k l} f_{j}=\eta_{i r} f_{s} f_{j} c_{i j k l} \sigma_{r s} .
$$

Renaming the indices $(r \rightarrow i, s \rightarrow j, i \rightarrow p, j \rightarrow q$ ) and inserting this result in equation (1) gives

$$
u_{n}(\mathbf{x})=\iint_{\Sigma} \sigma_{i j} N_{i j k l} G_{n k, l}(\mathbf{x}, \mathbf{s}) d^{2} s,
$$

with

$$
N_{i j k l}=\eta_{p i} f_{j} f_{q} c_{p q k l}
$$

[8] We assume that the properties of the fracture can be characterized by a normal compliance $\eta_{N}$ and a shear compliance $\eta_{T}$. In that case, one can use a dyadic decomposition to write the compliance matrix as $\eta=\eta_{N} \hat{\mathbf{f}} \hat{\mathbf{f}}^{T}+$ $\eta_{T}\left(\mathbf{I}-\hat{\mathbf{f}} \hat{\mathbf{f}}^{T}\right)$, where $\mathbf{I}$ is the identity matrix. This identity is, in component form, given by

$$
\eta_{i j}=\eta_{N} f_{i} f_{j}+\eta_{T}\left(\delta_{i j}-f_{i} f_{j}\right)
$$

where $\delta_{i j}$ is the Kronecker delta. We show in Appendix A that this compliance matrix in an isotropic medium gives

$$
N_{i j k l}=\lambda \eta_{N} f_{i} f_{j} \delta_{k l}+2 \mu\left(\eta_{N}-\eta_{T}\right) f_{i} f_{j} f_{k} f_{l}+\mu \eta_{T}\left(\delta_{i k} f_{j} f_{l}+\delta_{i l} f_{j} f_{k}\right),
$$

where $\lambda$ and $\mu$ are the Lamé parameters. Inserting equation (9) into equation (6) does not give the scattered waves because expression (6) constitutes an integral equation for the scattered field. (The stress $\sigma_{i j}$ in the integrand of equation (6) depends on the displacement field that we aim to compute.) We solve this integral equation in the Born approximation by replacing the stress in the right hand side of equation (6) by the stress $\sigma_{i j}^{(0)}$ for a $P$ or $S$ wave propagating through a homogeneous medium, depending on the type of incident wave. In that case the scattered wave is given by

$$
u_{n}(\mathbf{x})=\iint_{\Sigma} \sigma_{i j}^{(0)} N_{i j k l} G_{n k, l}(\mathbf{x}, \mathbf{s}) d^{2} s .
$$

Since $N_{i j k l}$ is known we can solve the scattering problem using the Born approximation. Replacing the stress field $\sigma_{i j}$ by the stress field $\sigma_{i j}^{(0)}$ of the incident wave is only valid when the perturbation of the stress state by the fracture is small. This is certainly not valid in the case of fluid-filled fractures, because for such fractures the shear traction vanishes at the fracture surface. For this reason the theory presented here is only applicable to dry fractures.

[9] Consider first an incoming plane $P$ wave that propagates in the $\hat{\mathbf{n}}$ direction (Figure 1). Since such a wave is polarized in the longitudinal direction,

$$
\mathbf{u}^{(P)}(\mathbf{s})=\hat{\mathbf{n}} e^{i k_{\alpha}(\hat{\mathbf{n}} \cdot \mathbf{s})}
$$




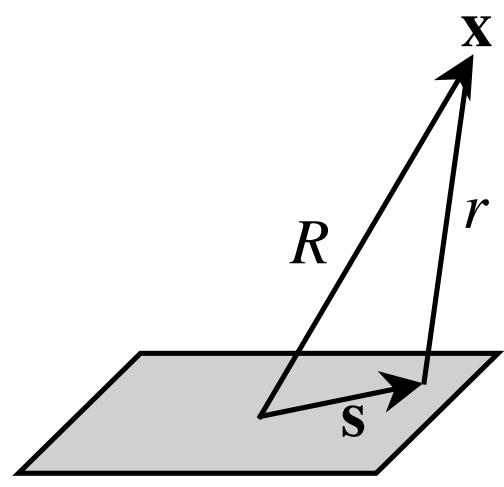

Figure 2. Definition of distance $R$ between the observation point $\mathbf{x}$ and the center of the fracture, and the distance $r$ between the observation point $\mathbf{x}$ and the integration point s on the fracture.

where

$$
k_{\alpha}=\omega / \alpha,
$$

with $\alpha$ the $P$ wave velocity and $\omega$ the angular frequency. For an isotropic medium $\sigma_{i j}=\lambda \delta_{i j} \partial_{k} u_{k}+\mu\left(\partial_{i} u_{j}+\partial_{j} u_{i}\right)$ and the stress associated with this plane $P$ wave is

$$
\sigma_{i j}^{(P)}=i k_{\alpha}\left(\lambda \delta_{i j}+2 \mu n_{i} n_{j}\right) e^{i k_{\alpha}(\hat{\mathbf{n}} \cdot \mathbf{s})} .
$$

For a plane $S$ wave arriving from the $\hat{\mathbf{n}}$ direction and polarized in the $\hat{\mathbf{p}}$ direction (Figure 1), the displacement is given by

$$
\mathbf{u}^{(S)}(\mathbf{s})=\hat{\mathbf{p}} e^{i k_{\beta}(\hat{\mathbf{n}} \cdot \mathbf{s})}
$$

where

$$
k_{\beta}=\omega / \beta,
$$

and $\beta$ is the $S$ wave velocity. The shear wave is transversely polarized, hence $(\hat{\mathbf{p}} \cdot \hat{\mathbf{n}})=0$. For an isotropic medium the associated stress is given by

$$
\sigma_{i j}^{(S)}=i k_{\beta} \mu\left(n_{i} p_{j}+n_{j} p_{i}\right) e^{i k_{\beta}(\hat{\mathbf{n}} \cdot \mathbf{s})} .
$$

Inserting the stress (13) or (16) into expression (10) gives the scattered field for incoming $P$ and $S$ waves, respectively.

\section{Scattering Amplitudes}

[10] The scattered field can effectively be expressed by a scattering amplitude [Merzbacher, 1970]. According to expression (10), the scattered field depends on $G_{n k, l}$, which is the gradient of the Green's function. Aki and Richards [2002, expression (4.29)] give the gradient of the Green's function in the time domain for a homogeneous, isotropic infinite space. Retaining the far field terms only and replacing the time derivative with $-i \omega$ gives, in the frequency domain

$$
G_{n k, l}(\mathbf{x}, \mathbf{s})=\frac{-i \omega m_{k} m_{n} m_{l}}{4 \pi \rho \alpha^{3} r} e^{i k_{\alpha} r}+\frac{-i \omega\left(\delta_{n k}-m_{k} m_{n}\right) m_{l}}{4 \pi \rho \beta^{3} r} e^{i k_{\beta} r}
$$

where the unit vector $\hat{\mathbf{m}}$ defines the direction of the outgoing wave (Figure 1) and $r=|\mathbf{x}-\mathbf{s}|$ denotes the distance between the observation point $\mathbf{x}$ and the integration point $\mathbf{s}$ on the fracture (Figure 2). In dyadic form, the term $\left(\delta_{n k}-m_{k} m_{n}\right)$ can be written as $\mathbf{I}-\hat{\mathbf{m}} \hat{\mathbf{m}}^{T}=\Sigma_{\mathrm{pol}} \hat{\mathbf{q}} \hat{\mathbf{q}}^{T}$, where $\hat{\mathbf{q}}$ is the polarization of the outgoing $S$ wave (Figure 1), and $\Sigma_{\text {pol }}$ represents the sum over the two orthogonal shear wave polarizations perpendicular to the direction of the outgoing wave. With this replacement, expression (17) can be written as

$$
G_{n k, l}(\mathbf{x}, \mathbf{s})=\frac{-i \omega m_{k} m_{n} m_{l}}{4 \pi \rho \alpha^{3} r} e^{i k_{\alpha} r}+\frac{-i \omega \sum_{\mathrm{pol}} q_{n} q_{k} m_{l}}{4 \pi \rho \beta^{3} r} e^{i k_{\beta} r} .
$$

[11] We choose the origin of our coordinate system near the center of the fracture, and denote the distance from the origin to the observation point by $R$ (Figure 2). When this distance is large compared to the size of the fracture, we can approximate

$$
r=R-(\hat{\mathbf{m}} \cdot \mathbf{s}),
$$

where $\hat{\mathbf{m}}$ is the unit vector from the center of the fracture to the observation point $\mathbf{x}$ (Figure 1), and $\mathbf{s}$ the location of the integration point on the fracture. Equation (18) varies most rapidly with $r$ through the exponents $e^{i k r}$. For this reason we replace $r$ by equation (19) in the exponents, and replace $r$ in the denominator by $R$. Inserting these results into equation (10) gives the following expressions for the radiated $P$ and $S$ waves

$$
u_{n}^{(P)}(\mathbf{x})=\iint_{\Sigma} \sigma_{i j}^{(0)} N_{i j k l} e^{-i k_{\alpha}(\hat{\mathbf{m}} \cdot \mathbf{s})} d^{2} s\left(\frac{-i \omega m_{n} m_{k} m_{l}}{4 \pi \rho \alpha^{3}}\right) \frac{e^{i k_{\alpha} R}}{R},
$$

$$
u_{n}^{(S)}(\mathbf{x})=\iint_{\Sigma} \sigma_{i j}^{(0)} N_{i j k l} e^{-i k_{\beta}(\hat{\mathbf{m}} \cdot \mathbf{s})} d^{2} s\left(\frac{-i \omega \sum_{\mathrm{pol}} q_{n} q_{k} m_{l}}{4 \pi \rho \beta^{3}}\right) \frac{e^{i k_{\beta} R}}{R} .
$$

In these expressions $\sigma_{i j}^{(0)}$ is given by equations (13) or (16) depending on whether the incoming wave is a $P$ wave or $S$ wave. We next define the scattering amplitude $f$ for outgoing $P$ and $S$ waves by

$$
\begin{gathered}
u_{n}^{(P)}(\mathbf{x})=f_{\cdot P} \frac{e^{i k_{\alpha} R}}{R} m_{n}, \\
u_{n}^{(S)}(\mathbf{x})=\sum_{\text {pol }} f_{\cdot S} \frac{e^{i k_{\beta} R}}{R} q_{n} .
\end{gathered}
$$

These equations are similar to the general expression of the scattering pattern in the far field for an heterogeneous inclusion, such as Martin [2006, equation (6.72)], see also Gubernatis et al. [1977a]. Note the presence of the polarization vectors for both types of waves $\left(m_{n}\right.$ and $q_{n}$, respectively). In the following $f_{P, P}$ is the scattering amplitude from a $P$ wave into a $P$ wave, $f_{S, P}$ is an $S$ to $P$ conversion, etc. Since the incoming wave in equations (22) and (23) can still be either a $P$ wave or an $S$ wave, we used the dot (.) in the first argument of the scattering amplitudes. A compari- 
son with equations (20) and (21) shows that the scattering amplitude is given by

$$
\begin{aligned}
& f_{\cdot P}=\iint_{\Sigma} \sigma_{i j}^{(0)} N_{i j k l} e^{-i k_{\alpha}(\hat{\mathbf{m}} \cdot \mathbf{s})} d^{2} s\left(\frac{-i \omega m_{k} m_{l}}{4 \pi \rho \alpha^{3}}\right), \\
& f_{\cdot S}=\iint_{\Sigma} \sigma_{i j}^{(0)} N_{i j k l} e^{-i k_{\beta}(\hat{\mathbf{m}} \cdot \mathbf{s})} d^{2} s\left(\frac{-i \omega q_{k} m_{l}}{4 \pi \rho \beta^{3}}\right) .
\end{aligned}
$$

[12] In the following expressions it is convenient to use a form factor $F(\mathbf{k})$ that is defined as

$$
F(\mathbf{k})=\frac{\iint_{\Sigma} e^{i(\mathbf{k} \cdot \mathbf{s})} d^{2} s}{\iint_{\Sigma} d^{2} s}=A^{-1} \iint_{\Sigma} e^{i(\mathbf{k} \cdot \mathbf{s})} d^{2} s,
$$

where $A$ is the surface area of the fracture. Explicit expressions for the scattering amplitude follow by inserting expressions (9) and (13) or (16) into the equations above. From here on the polarization of the outgoing $S$ wave is explicitly defined along $\hat{\mathbf{q}}$, as shown in Figure 1. As shown in Appendix B this gives the following scattering amplitudes for the different types of scattering

$$
\begin{aligned}
f_{P, P}(\hat{\mathbf{n}} ; \hat{\mathbf{m}})= & \frac{\omega^{2}}{4 \pi \rho \alpha^{4}} A F\left(k_{\alpha}(\hat{\mathbf{n}}-\hat{\mathbf{m}})\right) \\
& \times\left\{\lambda^{2} \eta_{N}+2 \lambda \mu \eta_{N}\left((\hat{\mathbf{n}} \cdot \hat{\mathbf{f}})^{2}+(\hat{\mathbf{m}} \cdot \hat{\mathbf{f}})^{2}\right)\right. \\
& +4 \mu^{2}\left(\eta_{N}-\eta_{T}\right)(\hat{\mathbf{n}} \cdot \hat{\mathbf{f}})^{2}(\hat{\mathbf{m}} \cdot \hat{\mathbf{f}})^{2} \\
& \left.+4 \mu^{2} \eta_{T}(\hat{\mathbf{n}} \cdot \hat{\mathbf{m}})(\hat{\mathbf{n}} \cdot \hat{\mathbf{f}})(\hat{\mathbf{m}} \cdot \hat{\mathbf{f}})\right\}
\end{aligned}
$$

$$
\begin{aligned}
f_{P, S}(\hat{\mathbf{n}} ; \hat{\mathbf{m}}, \hat{\mathbf{q}})= & \frac{\omega^{2}}{4 \pi \rho \alpha \beta^{3}} \mu A F\left(k_{\alpha} \hat{\mathbf{n}}-k_{\beta} \hat{\mathbf{m}}\right)\left\{2 \lambda \eta_{N}(\hat{\mathbf{m}} \cdot \hat{\mathbf{f}})(\hat{\mathbf{q}} \cdot \hat{\mathbf{f}})\right. \\
& +4 \mu\left(\eta_{N}-\eta_{T}\right)(\hat{\mathbf{n}} \cdot \hat{\mathbf{f}})^{2}(\hat{\mathbf{q}} \cdot \hat{\mathbf{f}})(\hat{\mathbf{m}} \cdot \hat{\mathbf{f}}) \\
& \left.+2 \mu \eta_{T}(\hat{\mathbf{n}} \cdot \hat{\mathbf{f}})((\hat{\mathbf{n}} \cdot \hat{\mathbf{q}})(\hat{\mathbf{m}} \cdot \hat{\mathbf{f}})+(\hat{\mathbf{n}} \cdot \hat{\mathbf{m}})(\hat{\mathbf{q}} \cdot \hat{\mathbf{f}}))\right\},
\end{aligned}
$$

$$
\begin{aligned}
f_{S, P}(\hat{\mathbf{n}}, \hat{\mathbf{p}} ; \hat{\mathbf{m}})= & \frac{\omega^{2}}{4 \pi \rho \alpha^{3} \beta} \mu A F\left(k_{\beta} \hat{\mathbf{n}}-k_{\alpha} \hat{\mathbf{m}}\right)\left\{2 \lambda \eta_{N}(\hat{\mathbf{n}} \cdot \hat{\mathbf{f}})(\hat{\mathbf{p}} \cdot \hat{\mathbf{f}})\right. \\
& +4 \mu\left(\eta_{N}-\eta_{T}\right)(\hat{\mathbf{n}} \cdot \hat{\mathbf{f}})(\hat{\mathbf{p}} \cdot \hat{\mathbf{f}})(\hat{\mathbf{m}} \cdot \hat{\mathbf{f}})^{2} \\
& \left.+2 \mu \eta_{T}(\hat{\mathbf{m}} \cdot \hat{\mathbf{f}})((\hat{\mathbf{p}} \cdot \hat{\mathbf{m}})(\hat{\mathbf{n}} \cdot \hat{\mathbf{f}})+(\hat{\mathbf{n}} \cdot \hat{\mathbf{m}})(\hat{\mathbf{p}} \cdot \hat{\mathbf{f}}))\right\},
\end{aligned}
$$

$$
\begin{aligned}
f_{S, S}(\hat{\mathbf{n}}, \hat{\mathbf{p}} ; \hat{\mathbf{m}}, \hat{\mathbf{q}}) & =\frac{\omega^{2}}{4 \pi \rho \beta^{4}} \mu^{2} A F\left(k_{\beta}(\hat{\mathbf{n}}-\hat{\mathbf{m}})\right) \\
& \times\left\{4\left(\eta_{N}-\eta_{T}\right)(\hat{\mathbf{n}} \cdot \hat{\mathbf{f}})(\hat{\mathbf{p}} \cdot \hat{\mathbf{f}})(\hat{\mathbf{m}} \cdot \hat{\mathbf{f}})(\hat{\mathbf{q}} \cdot \hat{\mathbf{f}})\right. \\
& +\eta_{T}(\hat{\mathbf{n}} \cdot \hat{\mathbf{q}})(\hat{\mathbf{p}} \cdot \hat{\mathbf{f}})(\hat{\mathbf{m}} \cdot \hat{\mathbf{f}})+\eta_{T}(\hat{\mathbf{n}} \cdot \hat{\mathbf{f}})(\hat{\mathbf{p}} \cdot \hat{\mathbf{q}})(\hat{\mathbf{m}} \cdot \hat{\mathbf{f}}) \\
& \left.+\eta_{T}(\hat{\mathbf{n}} \cdot \hat{\mathbf{m}})(\hat{\mathbf{p}} \cdot \hat{\mathbf{f}})(\hat{\mathbf{q}} \cdot \hat{\mathbf{f}})+\eta_{T}(\hat{\mathbf{n}} \cdot \hat{\mathbf{f}})(\hat{\mathbf{p}} \cdot \hat{\mathbf{m}})(\hat{\mathbf{q}} \cdot \hat{\mathbf{f}})\right\} .
\end{aligned}
$$

Note that the $P$ to $P$ scattering amplitude $f_{P, P}(\hat{\mathbf{n}} ; \hat{\mathbf{m}})$ depends only on the directions of incoming and outgoing waves, respectively, because these directions determine the polari- zation of the incoming and outgoing $P$ waves. In contrast, the $P$ to $S$ scattering amplitude $f_{P, S}(\hat{\mathbf{n}} ; \hat{\mathbf{m}}, \hat{\mathbf{q}})$ depends explicitly on the polarization of the outgoing $S$ wave as well. This dependence of the $S$ wave polarization appears whenever an $S$ wave is involved, either as incoming or outgoing wave. Expressions (27) through (30) do not change when $\hat{\mathbf{f}}$ is replaced by $-\hat{\mathbf{f}}$. This reflects the fact that both $\hat{\mathbf{f}}$ and $-\hat{\mathbf{f}}$ are normal to the fracture, and reversing the direction of the normal vector should not change the scattering of waves.

[13] For all incoming and outgoing waves in equations (27)-(30), the form factor (26) is evaluated at wave number $\mathbf{k}_{\text {in }}-\mathbf{k}_{\text {out }}$, where $\mathbf{k}_{\text {in }}$ is the wave number of the incoming wave and $\mathbf{k}_{\text {out }}$ that of the outgoing wave. It may appear that $F\left(\mathbf{k}_{\text {in }}-\mathbf{k}_{\text {out }}\right)$ violates reciprocity because it turns into its complex conjugate upon interchanging $\mathbf{k}_{\text {in }}$ and $\mathbf{k}_{\text {out }}$. Reciprocity is, however, not violated for the expressions of the scattered waves in expressions (22) and (23). The exponential in these expressions is given by $\exp \left(i \mathbf{k}_{\mathrm{out}} R\right)$, and the form factor contains another exponential $\exp \left(i\left(\mathbf{k}_{\text {in }}-\mathbf{k}_{\text {out }}\right) \cdot \mathbf{s}\right)$. The combination of the exponentials gives a total contribution $\exp \left(i \mathbf{k}_{\text {out }} R+i\left(\mathbf{k}_{\text {in }}-\mathbf{k}_{\text {out }}\right) \cdot \mathbf{s}\right)$. Using expression (19), and using that $\mathbf{k}_{\text {out }}=k_{\text {out }} \hat{\mathbf{m}}$, the phase is given by $k_{\text {out }} r+\mathbf{k}_{\text {out }}$. $\mathbf{s}+\left(\mathbf{k}_{\text {in }}-\mathbf{k}_{\text {out }}\right) \cdot \mathbf{s}=k_{\text {out }} r+\mathbf{k}_{\text {in }} \cdot \mathbf{s}$. This expression is the sum of the phase of the incident plane wave and the outgoing spherical wave for every integration point on the fracture, and the total scattered field obeys reciprocity.

\section{Scattering by a Plane Crack}

[14] We next derive explicit expressions for the scattering amplitudes in terms of the directions of the incoming and scattered waves for the special case of a plane crack that is either small or circular. We define a crack to be "small" when the argument $(\mathbf{k} \cdot \mathbf{s})$ in expression (26) is much smaller than 1 . This is the case when

$$
k_{\|} a \ll 1,
$$

where $k_{\|}$is the absolute value of the component of $\mathbf{k}$ parallel to the crack, and $a$ is the size of the crack. In equation (28) the form factor is given by $F\left(k_{\alpha} \hat{\mathbf{n}}-k_{\beta} \hat{\mathbf{m}}\right)$. The incoming $P$ wave has wave number $k_{\alpha} \hat{\mathbf{n}}$, while the outgoing scattered $S$ wave has wave number $k_{\beta} \hat{\mathbf{m}}$. The difference $k_{\alpha} \hat{\mathbf{n}}-k_{\beta} \hat{\mathbf{m}}$ thus denotes the change in the wave number during the scattering. In expressions (27)-(30) the form factor $F(\mathbf{k})$ is always evaluated at the wave number change during the scattering. Therefore, condition (31) does not necessarily imply that the fracture must be small compared to a wavelength. For example, for forward scattering of $P$ waves, $k_{\|}=$ $k_{\alpha}(\hat{\mathbf{n}}-\hat{\mathbf{m}})=0$ in expression (27), and condition (31) is satisfied for a fracture of any size. When condition (31) is satisfied, the exponent in equation (26) can be ignored and

$$
F(\mathbf{k})=1 \quad \text { (small fracture }) .
$$

We show in Appendix $\mathrm{C}$ that for a circular fracture with radius $a$

$$
F(\mathbf{k})=\frac{2}{k_{\|} a} J_{1}\left(k_{\|} a\right) \quad(\text { circular fracture }),
$$

where $J_{1}$ is the Bessel function of order 1. In the following we retain $F(\mathbf{k})$, but expressions (32) and (33) can be inserted 


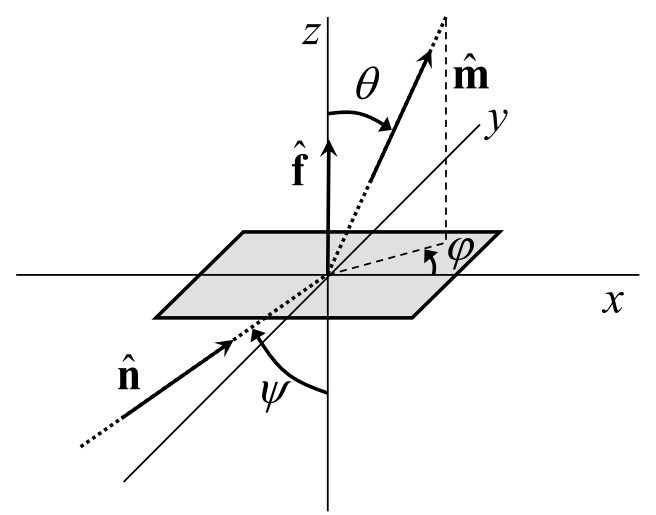

Figure 3. Definition of angles for incoming and outgoing waves from a fracture (shaded area).

for small cracks and circular cracks, respectively. According to Arfken and Weber [2001, expression (11.5)], $J_{1}(x)=x / 2+$ $O\left(x^{2}\right)$, hence expression (33) reduces to equation (32) for a small crack as $k_{\|} a \rightarrow 0$, and this holds independently of the incidence and scattering angles.

[15] In order to express the scattering amplitude in the angles that define the incoming and outgoing waves, we must define these angles and the orientation of the fracture. We use a coordinate system where the $z$ axis is perpendicular to the fracture, and the $x$ axis is chosen in such a way that the incoming wave propagates in the $(x, z)$ plane coming from the $-x$ direction (Figure 3). The direction of the incoming wave makes an angle $\psi$ with the $z$ axis, while the direction of the outgoing wave is defined by the angles $\theta$ and $\varphi$ that are commonly used in a spherical coordinate system. Referring to Figure 3 this means that the vector normal to the fracture and the directions of incoming and outgoing waves are given by

$$
\hat{\mathbf{f}}=\left(\begin{array}{l}
0 \\
0 \\
1
\end{array}\right), \quad \hat{\mathbf{n}}=\left(\begin{array}{c}
\sin \psi \\
0 \\
\cos \psi
\end{array}\right), \quad \hat{\mathbf{m}}=\left(\begin{array}{c}
\cos \varphi \sin \theta \\
\sin \varphi \sin \theta \\
\cos \theta
\end{array}\right)
$$

For a circular crack, these angles determine $k_{\|}$. For example, for $P$ to $S$ scattering it follows from expression (28), the definition of $k_{\|}$, and equation (34) that

$$
\begin{aligned}
k_{\| P, S} & =\left(k_{\alpha} \hat{\mathbf{n}}-k_{\beta} \hat{\mathbf{m}}\right)_{\|} \\
& =\sqrt{\left(k_{\alpha} \sin \psi-k_{\beta} \cos \varphi \sin \theta\right)^{2}+\left(k_{\beta} \sin \varphi \sin \theta\right)^{2}} .
\end{aligned}
$$

In the following we do not make this dependence on the angles explicit, but it should be kept in mind that for a circular crack one needs to account for the directions of incoming and outgoing waves in $F(\mathbf{k})$.

[16] We next specify the polarization vectors for shear waves. Using the terminology for layered media, we define a polarization vector $\hat{\mathbf{q}}^{S H}$ to be parallel to the fracture (Figure 4). Following Figures 3 and 4 the polarization vector for the $S H$ wave satisfies

$$
\hat{\mathbf{q}}^{S H}=\left(\begin{array}{c}
-\sin \varphi \\
\cos \varphi \\
0
\end{array}\right) .
$$

The other $S$ wave polarization $\left(\hat{\mathbf{q}}^{S V}\right)$ is oriented in the plane spanned by the normal vector $\mathbf{f}$ and the propagation direction $\hat{\mathbf{m}}$ (Figure 4), and is given by

$$
\hat{\mathbf{q}}^{S V}=\hat{\mathbf{m}} \times \hat{\mathbf{q}}^{S H}=\left(\begin{array}{c}
-\cos \varphi \cos \theta \\
-\sin \varphi \cos \theta \\
\sin \theta
\end{array}\right) .
$$

Since the fracture is finite, the label $S H$ should not be taken to mean that the shear wave with this polarization is decoupled from the $S V$ polarization and the $P$ waves. Indeed, the diffraction from the edges of the fracture contributes to nonzero scattering amplitudes $f_{S H, P}$ and $f_{S H, S V}$. The polarization vectors from incoming shear waves follow from expressions (36) and (37) by replacing $\varphi \rightarrow 0$ and $\theta \rightarrow \psi$, this gives

$$
\hat{\mathbf{p}}^{S H}=\left(\begin{array}{l}
0 \\
1 \\
0
\end{array}\right), \quad \hat{\mathbf{p}}^{S V}=\left(\begin{array}{c}
-\cos \psi \\
0 \\
\sin \psi
\end{array}\right) .
$$

[17] Inserting the direction vectors (34) and polarization vectors (36) and (37) into expressions (27) through (30) gives the angular dependence of the scattering amplitude. The scattering amplitude, which is different for the two $S$ polarizations, is given by

$$
\begin{aligned}
f_{P, P}(\hat{\mathbf{n}} ; \hat{\mathbf{m}})= & \frac{\omega^{2}}{4 \pi \rho \alpha^{4}} A F\left(k_{\alpha}(\hat{\mathbf{n}}-\hat{\mathbf{m}})\right) \\
& \times\left\{(\lambda+\mu)^{2} \eta_{N}+(\lambda+\mu) \mu \eta_{N}(\cos 2 \psi+\cos 2 \theta)\right. \\
& \left.+\mu^{2} \eta_{N} \cos 2 \psi \cos 2 \theta+\mu^{2} \eta_{T} \sin 2 \psi \sin 2 \theta \cos \varphi\right\}
\end{aligned}
$$

$$
\begin{aligned}
f_{P, S H}(\hat{\mathbf{n}} ; \hat{\mathbf{m}}, \hat{\mathbf{q}})= & \frac{\omega^{2}}{4 \pi \rho \alpha \beta^{3}} A F\left(k_{\alpha} \hat{\mathbf{n}}-k_{\beta} \hat{\mathbf{m}}\right) \\
& \times\left(-\mu^{2} \eta_{T}\right) \sin 2 \psi \cos \theta \sin \varphi
\end{aligned}
$$

$$
\begin{aligned}
f_{P, S V}(\hat{\mathbf{n}} ; \hat{\mathbf{m}}, \hat{\mathbf{q}})= & \frac{\omega^{2}}{4 \pi \rho \alpha \beta^{3}} A F\left(k_{\alpha} \hat{\mathbf{n}}-k_{\beta} \hat{\mathbf{m}}\right) \\
& \times\left\{(\lambda+\mu) \mu \eta_{N} \sin 2 \theta+\mu^{2} \eta_{N} \cos 2 \psi \sin 2 \theta\right. \\
& \left.-\mu^{2} \eta_{T} \sin 2 \psi \cos 2 \theta \cos \varphi\right\}
\end{aligned}
$$

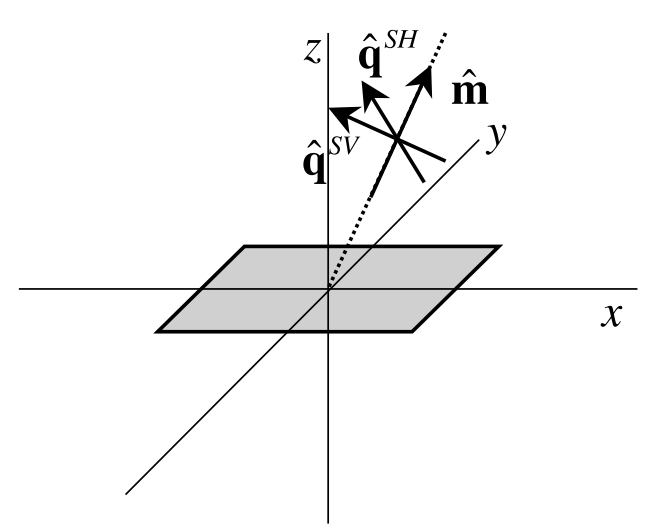

Figure 4. Polarization vectors for outgoing shear waves. 

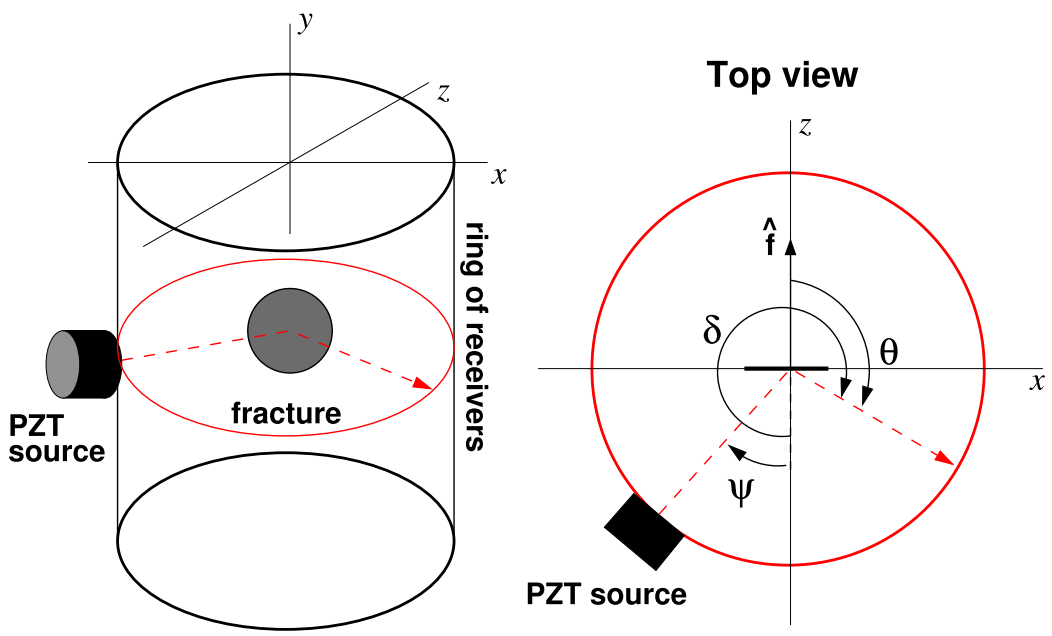

Figure 5. Geometry of the experimental setup with the angles as defined in Figure 3.

$$
f_{S H, P}(\hat{\mathbf{n}}, \hat{\mathbf{p}} ; \hat{\mathbf{m}})=\frac{\omega^{2}}{4 \pi \rho \alpha^{3} \beta} A F\left(k_{\beta} \hat{\mathbf{n}}-k_{\alpha} \hat{\mathbf{m}}\right) \mu^{2} \eta_{T} \cos \psi \sin 2 \theta \sin \varphi
$$

$$
\begin{aligned}
f_{S V, P}(\hat{\mathbf{n}}, \hat{\mathbf{p}} ; \hat{\mathbf{m}})= & \frac{\omega^{2}}{4 \pi \rho \alpha^{3} \beta} A F\left(k_{\beta} \hat{\mathbf{n}}-k_{\alpha} \hat{\mathbf{m}}\right) \\
& \times\left\{(\lambda+\mu) \mu \eta_{N} \sin 2 \psi+\mu^{2} \eta_{N} \sin 2 \psi \cos 2 \theta\right. \\
& \left.-\mu^{2} \eta_{T} \cos 2 \psi \sin 2 \theta \cos \varphi\right\}
\end{aligned}
$$

$f_{S H, S H}(\hat{\mathbf{n}}, \hat{\mathbf{p}} ; \hat{\mathbf{m}}, \hat{\mathbf{q}})=\frac{\omega^{2}}{4 \pi \rho \beta^{4}} A F\left(k_{\beta}(\hat{\mathbf{n}}-\hat{\mathbf{m}})\right) \mu^{2} \eta_{T} \cos \psi \cos \theta \cos \varphi$

$$
\begin{aligned}
f_{S H, S V}(\hat{\mathbf{n}}, \hat{\mathbf{p}} ; \hat{\mathbf{m}}, \hat{\mathbf{q}})= & \frac{\omega^{2}}{4 \pi \rho \beta^{4}} A F\left(k_{\beta}(\hat{\mathbf{n}}-\hat{\mathbf{m}})\right) \\
& \times\left(-\mu^{2} \eta_{T}\right) \cos \psi \cos 2 \theta \sin \varphi,
\end{aligned}
$$

$f_{S V, S H}(\hat{\mathbf{n}}, \hat{\mathbf{p}} ; \hat{\mathbf{m}}, \hat{\mathbf{q}})=\frac{\omega^{2}}{4 \pi \rho \beta^{4}} A F\left(k_{\beta}(\hat{\mathbf{n}}-\hat{\mathbf{m}})\right) \mu^{2} \eta_{T} \cos 2 \psi \cos \theta \sin \varphi$,

$$
\begin{aligned}
f_{S V, S V}(\hat{\mathbf{n}}, \hat{\mathbf{p}} ; \hat{\mathbf{m}}, \hat{\mathbf{q}})= & \frac{\omega^{2}}{4 \pi \rho \beta^{4}} A F\left(k_{\beta}(\hat{\mathbf{n}}-\hat{\mathbf{m}})\right)\left\{\mu^{2} \eta_{N} \sin 2 \psi \sin 2 \theta\right. \\
& \left.+\mu^{2} \eta_{T} \cos 2 \psi \cos 2 \theta \cos \varphi\right\}
\end{aligned}
$$

Expressions (27) through (30) each contain a contribution $4\left(\eta_{N}-\eta_{T}\right)$. In the derivation of equations (39) through (47) the contribution from the terms proportional to $4\left(\eta_{N}-\eta_{T}\right)$ is canceled by other terms containing $\eta_{T}$, which results in a considerable simplification of the resulting expressions.

[18] Note that any scattering coefficient with an $\mathrm{SH}$ wave as incoming or outgoing wave depends on $\mu$ and $\eta_{T}$, but not on $\lambda$ and $\eta_{N}$, which reflects that $S H$ waves do not depend on the compressive response of the medium. As a result, only the shear properties of the fracture influence the scattering to and from $\mathrm{SH}$ waves.

\section{Laboratory Experiments}

[19] We carry out laboratory experiments in order to measure $P$ to $P$ scattering and test our theoretical model. We use ultrasonic frequencies in plastic samples. The samples are Poly(methyl methacrylate) (PMMA) cylinders with a diameter of $50.8 \mathrm{~mm}$ and a height of $150 \mathrm{~mm}$ (Figure 5). Elastic waves are generated with a $5 \mathrm{MHz}$ disk-shaped piezoelectric transducer (PZT) with a diameter of $7.5 \mathrm{~mm}$, attached to the curved surface of the cylinder using phenyl salicylate as a glue. Because this glue has a melting point of $41.5^{\circ} \mathrm{C}$, slight heating is enough to melt it and use it to attach the transducer to a curved surface. The PZT is driven by a $400 \mathrm{~V}$ pulse with maximum energy at its natural frequency.

[20] We measure the elastic displacement with a laser interferometer. Our adaptive laser ultrasonic receiver is based on a doubled Nd:YAG laser, generating a Constant Wave (CW) $250 \mathrm{~mW}$ beam at a wavelength of $532 \mathrm{~nm}$. The receiver uses two-wave mixing in a photorefractive crystal to deliver the displacement of the sample surface. This receiver measures the out-of-plane (vertical) displacement field. It is calibrated to output the absolute displacement field in nanometers (see Blum et al. [2010] for a complete description). The frequency response is flat between $20 \mathrm{kHz}$ and $20 \mathrm{MHz}$, and it can accurately detect displacements of the order of parts of Angstroms. Since the sample material is transparent for green light, we apply a reflective tape to the surface to reflect light back to the laser receiver.

\subsection{Measurements on a Blank Sample}

[21] We first carry an experiment out on a blank cylinder. This measurement is used as a reference of the background field propagating in the absence of a scatterer. The sample is mounted on a computer-controlled rotational stage. We focus the laser receiver beam on the sample in a plane normal to the cylinder axis (taken as the $y$ axis). This plane 


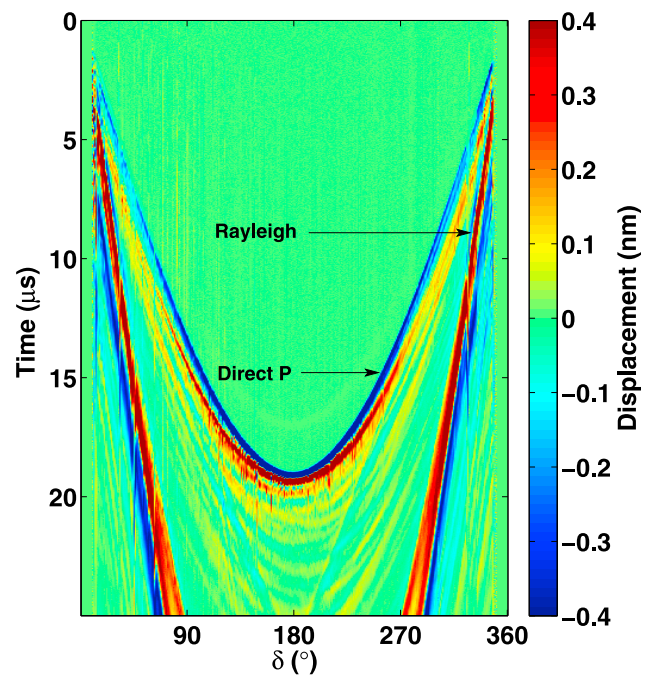

Figure 6. Displacement field for a homogeneous PMMA sample.

also contains the PZT source, the source and receiver are thus located in the $(x, z)$ plane. By computer-controlled rotation of the stage, we measure the elastic field in this plane every degree with respect to the center of the cylinder, except for a small range of angles blocked by the PZT source. The signal is digitized with 16-bit precision and a sampling rate of $100 \mathrm{MS} / \mathrm{s}$ (mega samples per second) and recorded on a computer acquisition board. For each receiver location, 256 waveforms are acquired and averaged after digitization.

[22] Figure 6 shows the raw ultrasonic displacement field for all recorded azimuths. The horizontal axis represents the angle $\delta$ between the source and the receiver directions, $\delta=\theta+180^{\circ}$ (for $\theta$ defined in Figure 3, see also Figure 5). The main events on this scan are the direct $P$ wave dis-

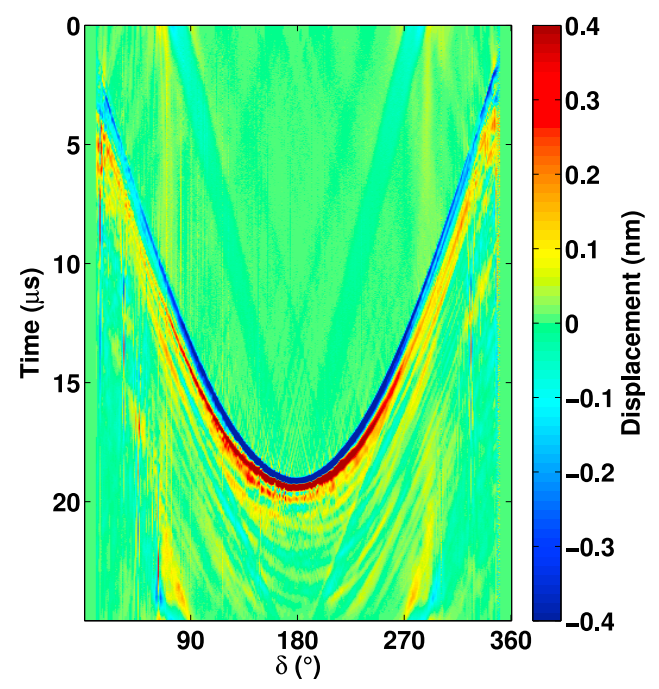

Figure 7. Displacement field for the homogeneous PMMA sample after $f-k$ filtering of the Rayleigh wave, highlighting the direct $P$ wave arrival. placement with a curved moveout and the Rayleigh wave traveling around the sample with a linear moveout. Some ringing of the source is present after the direct arrival and strongest for $\delta$ angles close to $180^{\circ}$. The frequency content of these data ranges from $250 \mathrm{kHz}$ to $1.2 \mathrm{MHz}$. In order to remove the high-amplitude Rayleigh wave arrival, we apply an $f-k$ filter to the data. The resulting displacement field is presented in Figure 7. All measurements following these are performed in the $(x, z)$ plane and $f-k$ filtered.

[23] From these data we find the $P$ and $S$ wave velocities of the material to be respectively $\alpha=2600 \mathrm{~m} / \mathrm{s}$ and $\beta=$ $1400 \mathrm{~m} / \mathrm{s}$. For a PMMA density of $\rho=1190 \mathrm{~kg} / \mathrm{m}^{3}$, these values correspond to Lamé coefficients $\lambda=3.4 \mathrm{GPa}$ and $\mu=$ $2.3 \mathrm{GPa}$, respectively.

\subsection{Fractured Sample}

[24] We create a single fracture in a different cylinder of PMMA by focusing a high power Q-switched Nd:YAG laser in the sample. The laser generates a short pulse ( $20 \mathrm{~ns})$ of infrared (IR) light that is absorbed by the sample material at the focal point and is converted into heat. The sudden thermal expansion generates stress and forms a fracture parallel to the cylindrical axis. Zadler and Scales [2008] give a more extensive description of the fracture generation process. The laser-generated fracture, shown in Figure 8, has a roughly circular shape and a radius of approximately $5 \mathrm{~mm}$. Figure 5 shows a diagram of the fractured sample and the geometry of the experimental setup.

[25] We show in Figure 9 the ultrasonic displacement after $f-k$ filtering, measured with the PZT source at location S1 normal to the fracture plane (Figure 10). This source location corresponds to an angle $\psi=0^{\circ}$. In addition to the events present with the blank sample, Figure 9 shows a wave arriving at about $20 \mu \mathrm{s}$, this arrival corresponds to the $P-P$

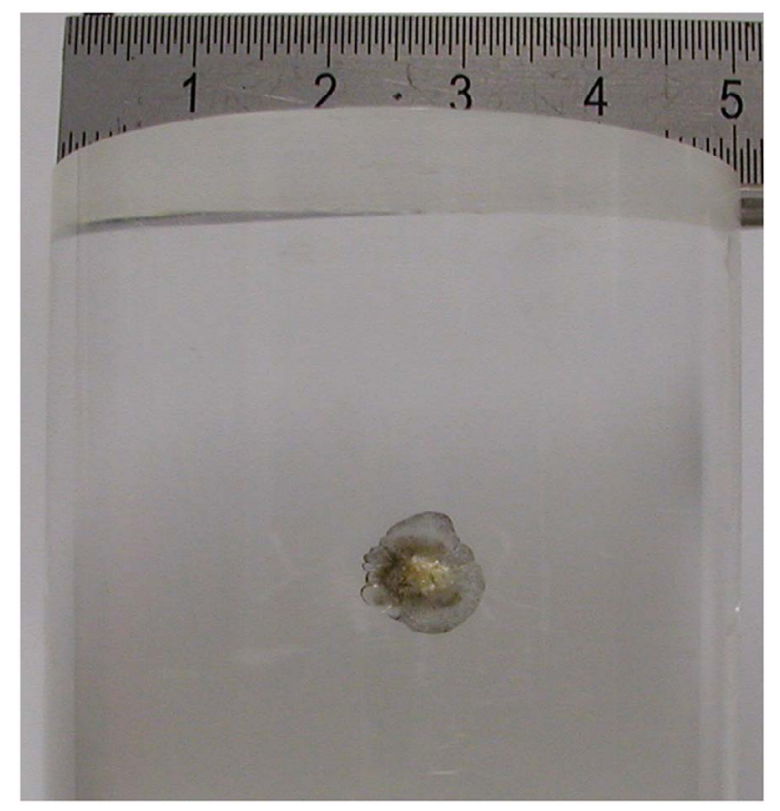

Figure 8. Photograph of the disk-shaped fracture in our laboratory sample. Ruler units are $\mathrm{cm}$. The diameter of the fracture is approximately $10 \mathrm{~mm}$, and the diameter of the cylinder is $50.8 \mathrm{~mm}$. 


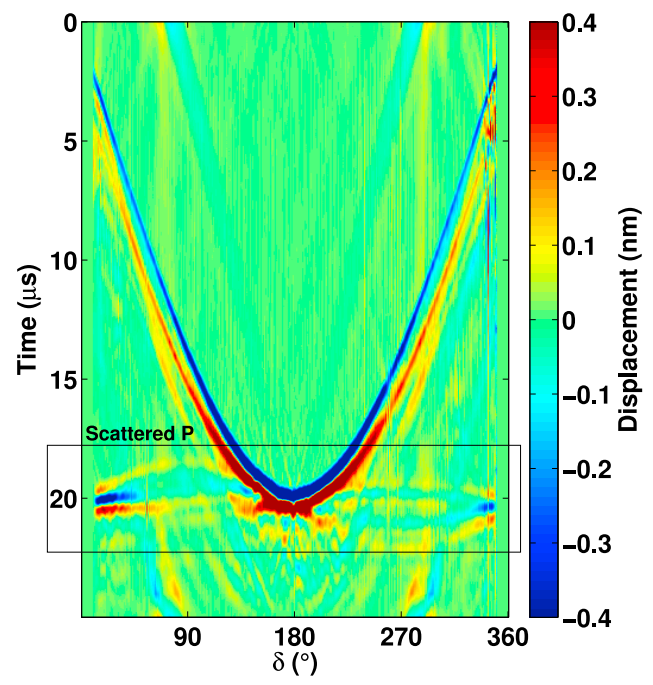

Figure 9. Displacement field for the fractured PMMA sample, with the source at normal incidence (after $f-k$ filtering).

scattered field from the crack. The amplitude of this event is maximum for $\delta=180^{\circ}$ (forward scattering), and $\delta=0^{\circ}$ (backscattering), corresponding to the specular reflection (Figure 10). Note that this event is slightly asymmetric: for receiver angles $\delta<180^{\circ}$, the scattering arrival is earlier than $20 \mu \mathrm{s}$, whereas for angles $\delta>180^{\circ}$, the wave arrives slightly later than $20 \mu \mathrm{s}$. This is due to the fact that the fracture is not perfectly centered on the $y$ axis. For forward scattering the scattered wave interferes with the direct wave (Figure 9), and the scattering amplitude cannot be measured accurately. The presence of the source transducer makes it difficult to measure the backscattered waves. For this reason Figure 12 does not show the scattering amplitude for scattering angles near forward and backward scattering.

[26] As we show in the next section, for this source position the scattering amplitude is a function of $\eta_{N}$ only. In an attempt to estimate $\eta_{T}$, we perform a last experiment with the PZT source at location S2, making an angle $\psi \approx 50^{\circ}$ to

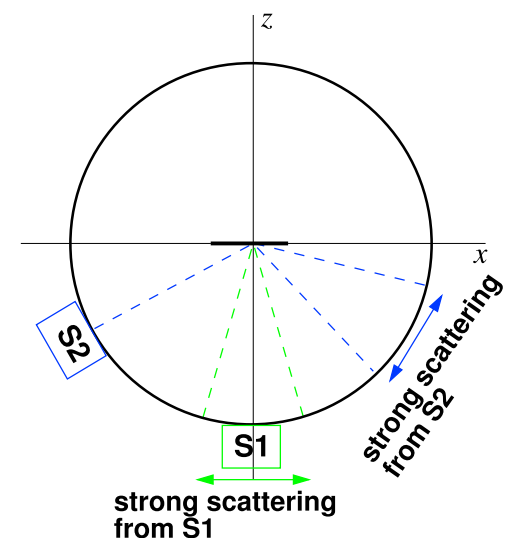

Figure 10. Schematic of the experimental setup with directions of maximum specular reflection for the two source positions. the normal to the fracture plane, but still in the $(x, z)$ plane (see Figure 9). The corresponding ultrasonic displacement field after $f-k$ filtering is shown in Figure 11. Note that, as shown in Figure 10, the scattering amplitude is largest for angles slightly larger than the specular reflection angle (corresponding to $\delta=310^{\circ}$ ).

\subsection{Scattering Amplitudes}

[27] The theoretical scattering amplitudes for all combinations of waves are given by expressions (39) through (47). Here the source is at a fixed angle $\psi$ to the normal of the fracture, hence $\hat{\mathbf{n}}=\sin \psi \hat{\mathbf{x}}+\cos \psi \hat{\mathbf{z}}$. The receiver is always in the $(x, z)$ plane, therefore $\phi=0^{\circ}$ and $\hat{\mathbf{m}}=\sin \theta \hat{\mathbf{x}}+\cos \theta \hat{\mathbf{z}}$.

[28] For the case of both incoming and scattered $P$ wave, equation (39) simplifies to

$$
\begin{aligned}
f_{P, P}(\hat{\mathbf{n}} ; \hat{\mathbf{m}})= & \frac{\omega^{2}}{4 \pi \rho \alpha^{4}} A F\left(k_{\alpha}(\hat{\mathbf{n}}-\hat{\mathbf{m}})\right) \\
& \times\left[\eta _ { N } \left((\lambda+\mu)^{2}+(\cos 2 \psi+\cos 2 \theta)(\lambda+\mu) \mu\right.\right. \\
& \left.\left.+\mu^{2}(\cos 2 \psi \cos 2 \theta)\right)+\eta_{T} \mu^{2}(\sin 2 \psi \sin 2 \theta)\right] .
\end{aligned}
$$

Moreover, for a circular fracture, equation (33) reduces for this geometry to

$$
F\left(k_{\alpha}(\hat{\mathbf{n}}-\hat{\mathbf{m}})\right) \approx \frac{2 \alpha}{a \omega(\sin \psi-\sin \theta)} J_{1}\left(\frac{\omega a}{\alpha}(\sin \psi-\sin \theta)\right) .
$$

[29] For the experimental case, the scattering amplitude is thus given by

$$
\begin{aligned}
f_{P, P}(\psi, \theta)= & \frac{\omega a}{2 \rho \alpha^{3}(\sin \psi-\sin \theta)} J_{1}\left(\frac{\omega a}{\alpha}(\sin \psi-\sin \theta)\right) \\
& \times\left[\eta _ { N } \left\{(\lambda+\mu)^{2}+(\cos 2 \psi+\cos 2 \theta)(\lambda+\mu) \mu\right.\right. \\
& \left.\left.+\mu^{2}(\cos 2 \psi \cos 2 \theta)\right\}+\eta_{T} \mu^{2}(\sin 2 \psi \sin 2 \theta)\right]
\end{aligned}
$$

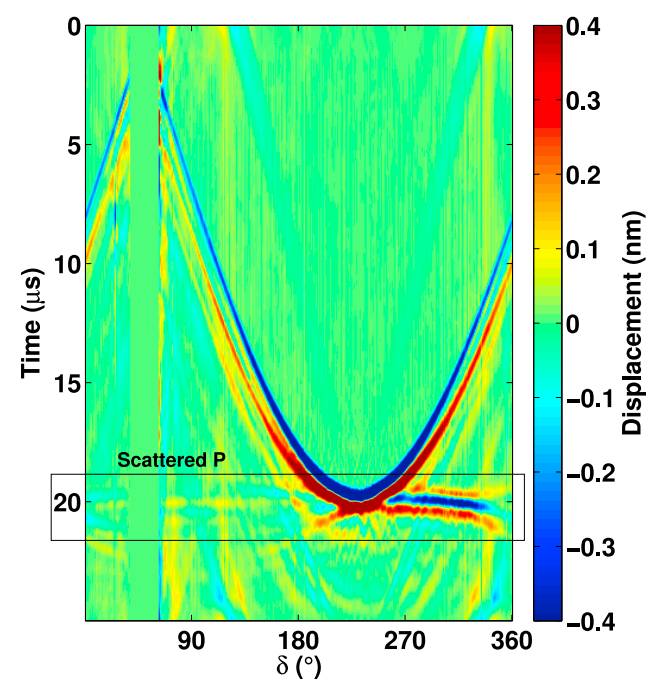

Figure 11. Displacement field for the fractured PMMA sample, where the source is at $\psi=50^{\circ}$ incidence (after $f-k$ filtering). 
Note that for a source at normal incidence, $\psi=0^{\circ}$ and therefore the term containing $\eta_{T}$ vanishes. In this case, the scattering amplitude $f_{P, P}\left(\psi=0^{\circ}, \theta\right)$ depends only on the normal component $\eta_{N}$ of the compliance tensor. On the other hand, for a nonnormal incidence $\psi$, the scattering amplitude $f_{P, P}$ is a function of both $\eta_{N}$ and $\eta_{T}$.

[30] To compare the experimental results with the analytic expression, we measure the scattering amplitude. We apply a narrow band-pass filter centered around $f_{0}=1 \mathrm{MHz}$, corresponding to the dominant frequency of the scattered event. We then pick the amplitude of the scattered arrival at its maximum for a range of angles excluding traces close to the source, and for receivers facing the source, where the incident and scattered field overlap. We normalize the scattering amplitude by the amplitude of the direct $P$ arrival at normal incidence, in order to compensate for differences in source coupling and strength between the two source locations. The experimental amplitudes for the valid range of angles are plotted in blue in Figures 12 and 13.

[31] We compute the corresponding theoretical amplitudes for $f_{0}=1 \mathrm{MHz}$, and use the Lamé coefficients computed from the measurement in the sample without fracture. We assume the created fracture behaves as a circular fracture with radius $a=5 \mathrm{~mm}$, estimated visually. We first optimize the fit with the theoretical amplitude (displayed in red) for the normal incidence data since for this angle of incidence the scattering amplitude depends only on normal component of the compliance $\eta_{N}$, but not on $\eta_{T}$. The best fit is obtained for $\eta_{N} \approx 10^{-11} \mathrm{~m} / \mathrm{Pa}$, corresponding to the thick dashed red curve in Figure 12. We also display the computed scattering amplitude for $\eta_{N}=2 \cdot 10^{-11} \mathrm{~m} / \mathrm{Pa}$ (dotted purple line) and $\eta_{N}=0.5 \cdot 10^{-11} \mathrm{~m} / \mathrm{Pa}$ (dotted orange line), to show that the $\eta_{N}=10^{-11} \mathrm{~m} / \mathrm{Pa}$ value is a robust fit. Note that the fit with $\eta_{N}$ only calibrates the overall amplitude of the scattering amplitude, but that the dependence of the scattering amplitude on the scattering angle is completely determined by the theory.

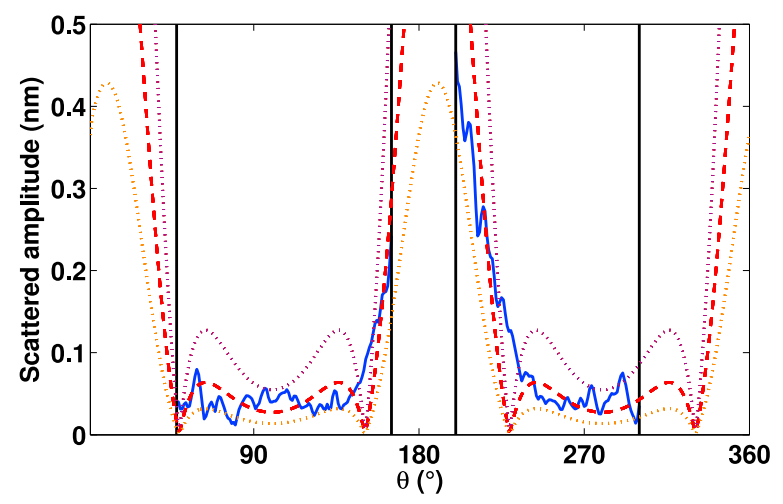

Figure 12. Scattering amplitude for the source at normal incidence in blue $\left(\psi=0^{\circ}\right)$. The best theoretical fit corresponding to $\eta_{N}=10^{-11} \mathrm{~m} / \mathrm{Pa}$ is plotted in thick dashed red. We also show the theoretical amplitudes corresponding to half (dotted orange) and twice (dotted purple) this value of $\eta_{N}$. The vertical black lines indicate the boundaries between regions where the scattered field is and is not measured.

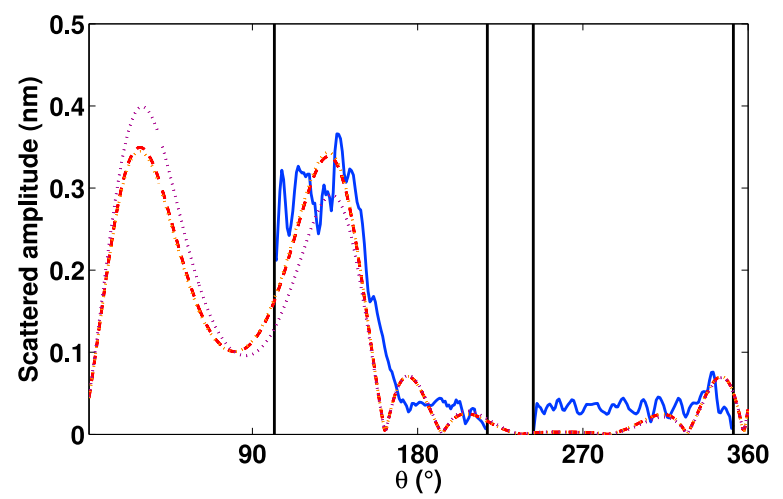

Figure 13. Scattering amplitude for the source at $\psi=50^{\circ}$ incidence in blue. The theoretical curve for $\eta_{N}=10^{-11} \mathrm{~m} / \mathrm{Pa}$ and $\eta_{T}=10^{-12} \mathrm{~m} / \mathrm{Pa}$ is plotted with a thick dashed red line. We also show the theoretical amplitudes corresponding to one tenth (dotted orange) and ten times (dotted purple) this value of $\eta_{T}$. We see here that the value of $\eta_{T}$ is not well constrained for this experimental configuration. The vertical black lines indicate the boundaries between regions where the scattered field is and is not measured.

[32] We then use this value for $\eta_{N}$ to optimize the fit of the second dataset by changing the shear compliance $\eta_{T}$. Figure 13 is a comparison between data and theoretical curves for $\eta_{N}=10^{-11} \mathrm{~m} / \mathrm{Pa}$ and three different values of the shear compliance: $\eta_{T}=10^{-12} \mathrm{~m} / \mathrm{Pa}$ (thick dashed red line) and $\eta_{T}=10^{-11} \mathrm{~m} / \mathrm{Pa}$ (dotted purple line) and $\eta_{T}=10^{-13} \mathrm{~m} / \mathrm{Pa}$ (dotted orange line). While according to equation (50) the scattering amplitude depends on the shear compliance $\eta_{T}$, this dependence is weak. The best fitting shear compliance $\eta_{T}=10^{-12} \mathrm{~m} / \mathrm{Pa}$ is an order of magnitude smaller than the estimated normal compliance, and the uncertainty in the estimate of the shear compliance ranges from $10^{-13} \mathrm{~m} / \mathrm{Pa}$ to $10^{-11} \mathrm{~m} / \mathrm{Pa}$. These values of compliances are, however, in the same range as $\eta \sim 10^{-13}-10^{-9} \mathrm{~m} / \mathrm{Pa}$ found in the literature for the case of a single fracture in steel [Pyrak-Nolte et al., 1990] and natural rocks [Worthington, 2007].

\section{Conclusions}

[33] Because fractures play a key role in processes going from seismic activity to fluid flow, fracture characterization is a critical step in time lapse monitoring of fluid flow in reservoirs. Based on a linear slip model, we derive the analytic expression of the scattered amplitude of a plane fracture of arbitrary size under the Born approximation. Of particular interest are the results for fractures of comparable size to the elastic wavelength. The theory provides scattering amplitudes for every combination of incident and scattered wave mode, which are expressed as a product of a Bessel function and trigonometric functions in the case of a circular fracture. Noncontacting ultrasonic data acquired on a plastic laboratory sample for $P$ wave to $P$ wave scattering from a circular fracture is in qualitative agreement with the theory, and the estimated compliance of the fracture agrees with the range of values reported in the literature. The theory presented here is not applicable to fluid-filled fractures, because the Born approximation used in equation (10) and 
subsequent expressions break down when the fluid in the fracture causes the shear traction at the fracture to vanish.

\section{Appendix A: Derivation of $N_{i j k l}$ for an Isotropic Medium}

[34] Inserting the expression for the elasticity tensor for an isotropic medium and equation (8) into definition (7) of $N_{i j k l}$ gives

$N_{i j k l}=\left\{\eta_{N} f_{p} f_{i}+\eta_{T}\left(\delta_{p i}-f_{p} f_{i}\right)\right\} f_{j} f_{q}\left\{\lambda \delta_{p q} \delta_{k l}+\mu \delta_{p k} \delta_{q l}+\mu \delta_{p l} \delta_{q k}\right\}$.

Carrying out the multiplication and summing over the variables of the delta functions gives

$$
\begin{aligned}
N_{i j k l}= & \lambda \delta_{k l}\left\{\eta_{N} f_{p} f_{i} f_{j} f_{p}+\eta_{T} f_{j} f_{i}-\eta_{T} f_{p} f_{i} f_{j} f_{p}\right\} \\
& +\mu\left\{\eta_{N} f_{k} f_{i} f_{j} f_{l}+\eta_{T} \delta_{i k} f_{j} f_{l}-\eta_{T} f_{k} f_{i} f_{j} f_{l}\right\} \\
& +\mu\left\{\eta_{N} f_{l} f_{i} f_{j} f_{k}+\eta_{T} \delta_{i l} f_{j} f_{k}-\eta_{T} f_{l} f_{i} f_{j} f_{k}\right\} .
\end{aligned}
$$

Since the vectors $\hat{\mathbf{f}}, \hat{\mathbf{n}}, \hat{\mathbf{p}}, \hat{\mathbf{m}}$ and $\hat{\mathbf{q}}$ are unit vectors

$$
f_{j} f_{j}=n_{j} n_{j}=p_{j} p_{j}=m_{j} m_{j}=q_{j} q_{j}=1 .
$$

Using this in equation (A2), and combining terms, leads to expression (9).

\section{Appendix B: Derivation of the Scattering Amplitude}

[35] In this appendix we show how to derive the scattering amplitudes in expressions (27)-(30). In order to derive $f_{P P}$, the stress (13) of an incoming $P$ wave and equation (9) combine to give

$$
\begin{aligned}
\sigma_{i j}^{(P)} N_{i j k l} m_{k} m_{l}= & i k_{\alpha} e^{i k_{\alpha}(\hat{\mathbf{n}} \mathbf{s})} \\
& \times\left\{\lambda^{2} \eta_{N} f_{i} f_{i} m_{k} m_{k}+2 \lambda \mu\left(\eta_{N}-\eta_{T}\right) f_{i} f_{i} f_{k} f_{l} m_{k} m_{l}\right. \\
& +\lambda \mu \eta_{T} f_{i} f_{i} m_{i} m_{l}+\lambda \mu \eta_{T} f_{i} f_{k} m_{k} m_{i} \\
& +2 \lambda \mu \eta_{N} n_{i} n_{j} f_{i} f_{j} m_{k} m_{k} \\
& +4 \mu^{2}\left(\eta_{N}-\eta_{T}\right) n_{i} n_{j} f_{i} f_{j} f_{k} f_{l} m_{k} m_{l} \\
& \left.+2 \mu^{2} \eta_{T} n_{i} n_{j} f_{j} f_{l} m_{i} m_{l}+2 \mu^{2} \eta_{T} n_{i} n_{j} f_{j} f_{k} m_{i} m_{k}\right\}
\end{aligned}
$$

Combinations, such as $n_{i} f_{i}$, are dot products and reduce to $(\hat{\mathbf{n}} \cdot \hat{\mathbf{f}})$. Using this, and the normalization (A3) in expression (B1), gives after combining terms

$$
\begin{aligned}
\sigma_{i j}^{(P)} N_{i j k l} m_{k} m_{l}= & i k_{\alpha} e^{i k_{\alpha}(\hat{\mathbf{n}} \cdot \mathbf{s})} \times\left\{\lambda^{2} \eta_{N}+2 \lambda \mu \eta_{N}\left((\hat{\mathbf{n}} \cdot \hat{\mathbf{f}})^{2}+(\hat{\mathbf{m}} \cdot \hat{\mathbf{f}})^{2}\right)\right. \\
& +4 \mu^{2}\left(\eta_{N}-\eta_{T}\right)(\hat{\mathbf{n}} \cdot \hat{\mathbf{f}})^{2}(\hat{\mathbf{m}} \cdot \hat{\mathbf{f}})^{2} \\
& \left.+4 \mu^{2} \eta_{T}(\hat{\mathbf{n}} \cdot \hat{\mathbf{m}})(\hat{\mathbf{n}} \cdot \hat{\mathbf{f}})(\hat{\mathbf{m}} \cdot \hat{\mathbf{f}})\right\} .
\end{aligned}
$$

Inserting this in equation (24), using that $k_{\alpha}=\omega / \alpha$ and definition (26) for $F(\mathbf{k})$, gives expression (27).

[36] Similar steps for $P$ to $S$ scattering give

$$
\begin{aligned}
\sigma_{i j}^{(P)} N_{i j k l} q_{k} m_{l}= & i k_{\alpha} e^{i k_{\alpha}(\hat{\mathbf{n}} \cdot \mathbf{s})} \times\left\{\lambda^{2} \eta_{N} f_{i} f_{i} q_{k} m_{k}+2 \lambda \mu \eta_{N} f_{i} f_{j} n_{i} n_{j} q_{k} m_{k}\right. \\
& +2 \lambda \mu\left(\eta_{N}-\eta_{T}\right) f_{i} f_{i} f_{k} f_{l} q_{k} m_{l} \\
& +4 \mu^{2}\left(\eta_{N}-\eta_{T}\right) n_{i} n_{j} f_{i} f_{j} f_{k} f_{l} q_{k} m_{l} \\
& +\lambda \mu \eta_{T} f_{i} f_{l} q_{i} m_{l}+2 \mu^{2} \eta_{T} n_{i} n_{j} f_{j} f_{l} q_{i} m_{l} \\
& \left.+\lambda \mu \eta_{T} f_{i} f_{k} q_{k} m_{i}+2 \mu^{2} \eta_{T} n_{i} n_{j} f_{j} f_{k} q_{k} m_{i}\right\} .
\end{aligned}
$$

The polarization of the outgoing $S$ wave is perpendicular to the direction of propagation, hence

$$
q_{k} m_{k}=(\hat{\mathbf{q}} \cdot \hat{\mathbf{m}})=0,
$$

hence the first two terms in the right hand side of expression (B3) vanish. Using this, and the normalization (A3), gives

$$
\begin{aligned}
\sigma_{i j}^{(P)} N_{i j k l} q_{k} m_{l}= & i k_{\alpha} e^{i k_{\alpha}(\hat{\mathbf{n}} \mathbf{s})} \times\left\{2 \lambda \mu \eta_{N}(\hat{\mathbf{m}} \cdot \hat{\mathbf{f}})(\hat{\mathbf{q}} \cdot \hat{\mathbf{f}})\right. \\
& +4 \mu^{2}\left(\eta_{N}-\eta_{T}\right)(\hat{\mathbf{n}} \cdot \hat{\mathbf{f}})^{2}(\hat{\mathbf{m}} \cdot \hat{\mathbf{f}})(\hat{\mathbf{q}} \cdot \hat{\mathbf{f}}) \\
& \left.+2 \mu^{2} \eta_{T}(\hat{\mathbf{n}} \cdot \hat{\mathbf{f}})(\hat{\mathbf{m}} \cdot \hat{\mathbf{f}})((\hat{\mathbf{n}} \cdot \hat{\mathbf{q}})+(\hat{\mathbf{n}} \cdot \hat{\mathbf{m}}))\right\} .
\end{aligned}
$$

Using this in expression (25) leads, with definition (26), to equation (28).

[37] For $S$ to $P$ scattering we use expression (16) for the stress, hence

$$
\begin{aligned}
\sigma_{i j}^{(S)} N_{i j k l} m_{k} m_{l}= & i k_{\beta} e^{i k_{\beta}(\hat{\mathbf{n}} \cdot \mathbf{s})} \\
& \times\left\{\lambda \mu \eta_{N} n_{i} p_{j} f_{i} f_{j} m_{k} m_{k}+\lambda \mu \eta_{N} n_{j} p_{i} f_{i} f_{j} m_{k} m_{k}\right. \\
& +2 \mu^{2}\left(\eta_{N}-\eta_{T}\right) n_{i} p_{j} f_{i} f_{j} f_{k} f f_{l} m_{k} m_{l} \\
& +2 \mu^{2}\left(\eta_{N}-\eta_{T}\right) n_{j} p_{i} f_{i} f_{j} f_{k} f_{l} m_{k} m_{l} \\
& +\mu^{2} \eta_{T} n_{i} p_{j} f_{j} f_{l} m_{i} m_{l}+\mu^{2} \eta_{T} n_{j} p_{i} f_{j} f_{l} m_{i} m_{l} \\
& \left.+\mu^{2} \eta_{T} n_{i} p_{j} f_{j} f_{j} m_{i} m_{k}+\mu^{2} \eta_{T} n_{j} p_{i} f_{j} f_{k} m_{i} m_{k}\right\}
\end{aligned}
$$

Using expression (A3) this can be reorganized as

$$
\begin{aligned}
\sigma_{i j}^{(S)} N_{i j k l} m_{k} m_{l}= & i k_{\beta} e^{i k_{\beta}(\hat{\mathbf{n}} \cdot \mathbf{s})} \times\left\{2 \lambda \mu \eta_{N}(\hat{\mathbf{n}} \cdot \hat{\mathbf{f}})(\hat{\mathbf{p}} \cdot \hat{\mathbf{f}})\right. \\
& +4 \mu^{2}\left(\eta_{N}-\eta_{T}\right)(\hat{\mathbf{n}} \cdot \hat{\mathbf{f}})(\hat{\mathbf{p}} \cdot \hat{\mathbf{f}})(\hat{\mathbf{m}} \cdot \hat{\mathbf{f}})^{2} \\
& \left.+2 \mu^{2} \eta_{T}(\hat{\mathbf{m}} \cdot \hat{\mathbf{f}})((\hat{\mathbf{n}} \cdot \hat{\mathbf{m}})(\hat{\mathbf{p}} \cdot \hat{\mathbf{f}})+(\hat{\mathbf{p}} \cdot \hat{\mathbf{m}})(\hat{\mathbf{n}} \cdot \hat{\mathbf{f}}))\right\}
\end{aligned}
$$

Inserting this in equation (24) leads with expression (26) to the $S$ to $P$ scattering amplitude (29).

[38] Finally the $S$ to $S$ scattering amplitude follows from the same steps:

$$
\begin{aligned}
\sigma_{i j}^{(S)} N_{i j k l} q_{k} m_{l}= & i k_{\beta} e^{i k_{\beta}(\hat{\mathbf{n}} \cdot \mathbf{s})} \\
& \times\left\{\lambda^{2} \mu \eta_{N} n_{i} p_{j} f_{i} f_{j} q_{k} m_{k}+\lambda^{2} \mu \eta_{N} n_{j} p_{i} f_{i} f_{j} q_{k} m_{k}\right. \\
& +2 \mu^{2}\left(\eta_{N}-\eta_{T}\right)\left(n_{i} p_{j} f_{i} f_{j} f_{k} f_{l} q_{k} m_{l}+n_{j} p_{i} f_{i} f_{j} f_{k} f_{l} q_{k} m_{l}\right) \\
& +\mu^{2} \eta_{T} n_{i} p_{j} f_{j} f_{l} q_{i} m_{l}+\mu^{2} \eta_{T} n_{j} p_{i} f_{j} f_{l} q_{i} m_{l} \\
& \left.+\mu^{2} \eta_{T} n_{i} p_{j} f_{j} f_{k} q_{k} m_{i}+\mu^{2} \eta_{T} n_{j} p_{i} f_{j} f_{k} q_{k} m_{i}\right\} .
\end{aligned}
$$

The polarization vector $\hat{\mathbf{q}}$ of the outgoing $S$ wave is perpendicular to the direction of propagation, hence $q_{k} m_{k}=$ $(\hat{\mathbf{m}} \cdot \hat{\mathbf{q}})=0$, and the terms proportional to $\lambda$ vanish. The remaining terms are, in vector notation, given by

$$
\begin{aligned}
\sigma_{i j}^{(S)} N_{i j k l} q_{k} m_{l}= & i k_{\beta} e^{i k_{k}(\hat{\mathbf{n}} \mathbf{s})} \mu^{2} \\
& \times\left\{4\left(\eta_{N}-\eta_{T}\right)(\hat{\mathbf{n}} \cdot \hat{\mathbf{f}})(\hat{\mathbf{p}} \cdot \hat{\mathbf{f}})(\hat{\mathbf{m}} \cdot \hat{\mathbf{f}})(\hat{\mathbf{q}} \cdot \hat{\mathbf{f}})\right. \\
& +\eta_{T}(\hat{\mathbf{n}} \cdot \hat{\mathbf{q}})(\hat{\mathbf{p}} \cdot \hat{\mathbf{f}})(\hat{\mathbf{m}} \cdot \hat{\mathbf{f}})+\eta_{T}(\hat{\mathbf{n}} \cdot \hat{\mathbf{f}})(\hat{\mathbf{p}} \cdot \hat{\mathbf{q}})(\hat{\mathbf{m}} \cdot \hat{\mathbf{f}}) \\
& \left.+\eta_{T}(\hat{\mathbf{n}} \cdot \hat{\mathbf{m}})(\hat{\mathbf{p}} \cdot \hat{\mathbf{f}})(\hat{\mathbf{q}} \cdot \hat{\mathbf{f}})+\eta_{T}(\hat{\mathbf{n}} \cdot \hat{\mathbf{f}})(\hat{\mathbf{p}} \cdot \hat{\mathbf{m}})(\hat{\mathbf{q}} \cdot \hat{\mathbf{f}})\right\}
\end{aligned}
$$


Using this, and definition (26), in expression (25) gives equation (30).

\section{Appendix C: $\boldsymbol{F}(\mathbf{k})$ for a Circular Crack}

[39] Following definition (26), the form factor for a circular crack with radius $a$ is given by

$$
F(\mathbf{k})=A^{-1} \iint_{\Sigma} e^{i(\mathbf{k} \cdot \mathbf{s})} d^{2} s=A^{-1} \int_{0}^{a} \int_{0}^{2 \pi} e^{i k_{\|} s \cos \xi} d \xi s d s
$$

where $\xi$ is the angle between the projection of $\mathbf{k}$ on the fracture and the integration variable $\mathbf{s}$. The integral representation of the Bessel function as given by Arfken and Weber [2001, expression (11.30c)] $\left(2 \pi J_{0}(x)=\int_{0}^{2 \pi} e^{i x \cos \xi} d \xi\right)$ reduces expression $(\mathrm{C} 1)$ to

$$
F(\mathbf{k})=A^{-1} \iint_{\Sigma} e^{i(\mathbf{k} \cdot \mathbf{s})} d^{2} s=\frac{2 \pi}{A} \int_{0}^{a} s J_{0}\left(k_{\|} s\right) d s,
$$

where $J_{0}$ is the Bessel function of order zero. We next use the recursive relation $d\left(x^{n} J_{n}(x)\right) / d x=x^{n} J_{n-1}(x)$ [Arfken and Weber, 2001, equation (11.15)]. Setting $n=1$ and $x=k_{\|} S$ gives

$$
s J_{0}\left(k_{\|} s\right)=k_{\|}^{-1} \frac{d}{d s}\left(s J_{1}\left(k_{\|} s\right)\right) .
$$

Inserting this in expression (C2) yields

$$
F(\mathbf{k})=\frac{2 \pi}{k_{\|} A} \int_{0}^{a} \frac{d}{d s}\left(s J_{1}\left(k_{\|} s\right)\right) d s=\frac{2 \pi}{k_{\|} A} a J_{1}\left(k_{\|} a\right) .
$$

Using that $A=\pi a^{2}$ gives equation (33).

[40] Acknowledgments. We thank ConocoPhillips, especially Phil Anno, for supporting this research. We also thank John Scales and Filippo Broggini from the Colorado School of Mines and fellow members of the Physical Acoustics Laboratory at Boise State University for their constructive ideas and comments.

\section{References}

Aki, K., and P. G. Richards (2002), Quantitative Seismology, 2nd ed., Univ. Sci. Books, Sausalito, Calif.

Arfken, G. B., and H. Weber (2001), Mathematical Methods for Physicists, 5th ed., Harcourt, Amsterdam.

Blum, T. E., K. van Wijk, B. Pouet, and A. Wartelle (2010), Multicomponent wavefield characterization with a novel scanning laser interferometer, Rev. Sci. Instrum., 81(7), 073101, doi:10.1063/1.3455213.

Brandsdóttir, B., and P. Einarsson (1979), Seismic activity associated with the September 1977 deflation of the Krafla central volcano in northeastern Iceland, J. Volcanol. Geotherm. Res., 6(3-4), 197-212, doi:10.1016/ 0377-0273(79)90001-5.

Coates, R. T. and M. A. Schoenberg (1995), Finite-difference modeling of faults and fractures, Geophysics, 60, 1514-1526, doi:10.1190/1.1443884.

Collettini, C., A. Niemeijer, C. Viti, and C. Marone (2009), Fault zone fabric and fault weakness, Nature, 462, 907-911.

Crampin, S. (1981), A review of wave motion in anisotropic and cracked elastic media, Wave Motion, 3, 343-391, doi:10.1016/0165-2125(81) 90026-3

Fang, X., M. Fehler, T. Chen, and D. R. Burns (2010), Sensitivity analysis of fracture scattering, SEG Tech. Program Expanded Abstr., 29(1), 2340-2345, doi:10.1190/1.3513320.

Groenenboom, J., and J. Falk (2000), Scattering by hydraulic fractures: Finite-difference modeling and laboratory data, Geophysics, 65, 612-622, doi:10.1190/1.1444756.

Groenenboom, J., and J. T. Fokkema (1998), Monitoring the width of hydraulic fractures with acoustic waves, Geophysics, 63, 139-148.

Groenenboom, J., and D. van Dam (2000), Monitoring hydraulic fracture growth: Laboratory experiments, Geophysics, 65, 603-611.
Gubernatis, J., E. Domany, and J. Krumhansl (1977a), Formal aspects of the theory of scattering of ultrasound by flaws in elastic materials, J. Appl. Phys., 48, 2804-2811.

Gubernatis, J., E. Domany, J. Krumhansl, and M. Huberman (1977b), The Born approximation in the theory of the scattering of elastic waves by cracks, J. Appl. Phys., 48, 2812-2819.

Haney, M. M., R. Snieder, J. Sheiman, and S. Losh (2005), Geophysics: A moving fluid pulse in a fault zone, Nature, 437(7055), 46, doi:10.1038/ 437046a.

Hudson, J. A. (1981), Wave speeds and attenuation of elastic waves in materials containing cracks, Geophys. J. R. Astron. Soc., 64, 133-150, doi:10.1111/j.1365-246X.1981.tb02662.x.

Kachanov, M., and I. Sevostianov (2005), On quantitative characterization of microstructures and effective properties, Int. J. Solids Struct., 42, 309-336, doi:10.1016/j.ijsolstr.2004.06.016.

Langenberg, K., R. Marklein, and K. Mayer (2002), Applications to nondestructive testing with ultrasound, in Scattering and Inverse Scattering in Pure and Applied Science, edited by P. Sabatier and E. Pike, pp. 594-617, Academic, San Diego, Calif.

Martin, P. (2006), Multiple Scattering, Encyclopedia of Mathematics and Its Applications, vol. 107, 437 pp., Cambridge Univ. Press, Cambridge, U. K.

Meadows, M. A., and D. Winterstein (1994), Seismic detection of a hydraulic fracture from shear wave VSP data at Lost Hills Field, California, Geophysics, 59, 11-26, doi:10.1190/1.1443523

Merzbacher, E. (1970), Quantum Mechanics, 2nd ed., John Wiley, New York.

Pyrak-Nolte, L. J. (2000), Monitoring fracture evolution with compressional-mode interface waves, J. Geophys. Res., 27, 3397-3400.

Pyrak-Nolte, L. J., and D. D. Nolte (1992), Frequency dependence of fracture stiffness, Geophys. Res. Lett., 19, 325-328, doi:199210.1029 91GL03179.

Pyrak-Nolte, L. J., L. Myer, and N. Cook (1990), Transmission of seismic waves across a single natural fracture, J. Geophys. Res., 95, 8617-8638, doi:199010.1029/JB095iB06p08617.

Pyrak-Nolte, L. J., J. Xu, and G. Haley (1992), Elastic interface waves along a fracture: Theory and experiment, in Proceedings of the 33rd U.S. Rock. Mechanics Symposeum, edited by J. R. Tillerson and W. R. Wawersik, pp. 999-1007, AA Balkema, Rotterdam, Netherlands.

Pyrak-Nolte, L. J., S. Roy, and B. Mullenbach (1996), Interface waves propagated along a fracture, J. Appl. Geophys., 35, 79-87.

Sánchez-Sesma, F. J., and U. Iturrarán-Viveros (2001), Scattering and diffraction by SH waves by a finite crack: An analytical solution, Geophys. J. Int., 145, 749-758, doi:10.1046/j.1365-246x.2001.01426.x.

Schoenberg, M. A. (1980), Elastic wave behavior across linear slip interfaces, J. Acoust. Soc. Am., 68, 1516-1521, doi:10.1121/1.385077.

Schoenberg, M. A., and J. Douma (1988), Elastic wave propagation in media with parallel fractures and aligned cracks, Geophys. Prospect., 36, 571-590, doi:10.1111/j.1365-2478.1988.tb02181.x.

Schoenberg, M. A., and C. Sayers (1995), Seismic anisotropy of fractured rock, Geophysics, 60, 204-211, doi:10.1190/1.1443748.

Smyshlyaev, V., and J. Willis (1994), Linear ad nonlinear scattering of elastic waves by microcracks, J. Mech. Phys. Solids, 42, 585-610.

Wills, P., D. DeMartini, H. Vinegar, J. Shlyapobersky, W. Deeg, J. Woerpel, J. Fix, G. Sorrelis, and R. Adair (1992), Active and passive imaging of hydraulic fractures, Leading Edge, 11, 15-22, doi:10.1190/1.1436884.

Worthington, M. (2007), The compliance of macrofractures, Leading Edge, 26, 1118-1121.

Wu, C., J. M. Harris, K. T. Nihei, and S. Nakagawa (2005), Twodimensional finite-difference seismic modeling of an open fluid-filled fracture: Comparison of thin-layer and linear-slip models, Geophysics, 70, T57, doi:10.1190/1.1988187

Wu, R., and K. Aki (1985), Scattering characteristics of elastic waves by an elastic heterogeneity, Geophysics, 50, 582-595.

Zadler, B. J., and J. A. Scales (2008), Monitoring crack-induced changes in elasticity with resonant spectroscopy, J. Appl. Phys., 104(2), 023536, doi: $10.1063 / 1.2956688$

Zhu, Y., and R. Snieder (2002), Reflected and transmitted waves from fault zones, SEG Tech. Program Expanded Abstr., 21(1), 2273-2276, doi:10.1190/1.1817166.

T. E. Blum and K. van Wijk, Physical Acoustics Laboratory, Department of Geosciences, Boise State University, 1910 University Dr., Boise, ID 83725, USA. (tblum@cgiss.boisestate.edu; kaspervanwijk@boisestate.edu) R. Snieder, Center for Wave Phenomena, Colorado School of Mines, 1500 Illinois St., Golden, CO 80401, USA. (rsnieder@mines.edu)

M. E. Willis, ConocoPhillips Company, 2056 Permian, 600 North Dairy Ashford, Houston, TX 77079, USA. (mark e willis@hotmail.com) 